A la fin du mois de mars de cette année, le Prof. Christoph Rehmann-Sutter a démissionné de ses fonctions, après 8 ans comme président de la Commission nationale d'éthique dans le domaine de la médecine humaine (CNE). A cette occasion, le 23 avril, un congrès d'adieu s'est tenu à Berne, sur le thème «Gesundheit als öffentliche Aufgabe ethische Perspektiven» (Ia santé comme mission publique; perspectives éthiques): lors de cette mani- festation, I'ancien président de la CNE a posé la question de savoir si la santé est (aussi) une «affaire publique», dans quelle mesure et quelles fonctions une commission nationale d'éthique peut remplir dans ce contexte. Christoph Rehmann-Sutter présente ci-après une version abrégée de son intervention, sous forme $d^{\prime}$ article.*

La rédaction

\title{
Wie weit reicht die öffentliche Verantwortung für Gesundheit?
}

\section{Christoph Rehmann-Sutter}

* Lesen Sie hierzu auch «Zu guter Letzt» von Jean Martin auf Seite 1312
Korrespondenz:

Prof. Dr. phil., dipl. biol. Christoph Rehmann-Sutter

Institut für Medizingeschichte und Wissenschaftsforschung der Universität zu Lübeck Königstrasse 4

D-23552 Lübeck

Tel. 0049 (0)451 70799812 Fax 0049 (0)451 70799899

rehmann@

imgwf.uni-luebeck.de
Wissen wir denn so genau, was Gesundheit ist, um sie gesellschaftlich herstellen zu können? Der Philosoph Hans-Georg Gadamer hat (in hohem Alter) davon gesprochen, dass die Krankheiten auffallen, während uns die Gesundheit verborgen bleibt. Die berühmte Definition von Gesundheit in der WHO-Verfassung von 1948 spricht von einem Zustand vollständigen Wohlbefindens: «Health is a state of complete physical, mental and social well-being and not merely the absence of disease or infirmity.» Das ist natürlich ein Idealbegriff. Er spricht nicht in positiver Form, sondern nur in negativer Form davon, was die Gesundheit zusätzlich zur Abwesenheit von Krankheit ausmachen soll: «... not merely the absence of ...». Die erfahrene Gesundheit muss aber konkreter sein als das vollständige Wohlbefinden. Sie scheint weitaus komplexer zu sein niemanden sonst etwas angeht, schon gar nicht den Staat. Wenn es ein anerkannter Teil des Selbstbestimmungsrechts ist, über die Art und Weise des eigenen Todes selbst zu bestimmen - wie es das Bundesgericht vorletztes Jahr im Urteil zur Suizidbeihilfe bestätigte dann ist es doch erst recht Teil des Selbstbestimmungsrechts, darüber zu befinden, welche Therapien man akzeptieren möchte, wenn man krank geworden ist, und überhaupt selbst zu beurteilen, was für einen als gesund und was als krank gilt. Nicht für alle gelten die gleichen Zustände als «gesund». Und wir sehen auch Sinn und Sinnlosigkeit von Krankheiten, das heisst auch die Sinn-Brüche, die Konflikte, die Grenzsituationen je individuell. Diesen Sinn auszulegen und immer wieder neu daran zu arbeiten, gehört zu unserer Subjektivität.

\section{«Public health» ist ein bemerkenswerter Begriff: als ob die Öffentlichkeit gesund sein könnte, oder das Öffentliche. Gesund und krank im eigentlichen Sinn sind die Individuen»}

als ein Ideal, und vor allem enthält sie Ambivalenzen. Jemand kann chronisch krank sein und sich doch heute gesund fühlen. Oder jemand kann sehr gesund mit einem psychischen Leiden umgehen. Die Erinnerung an die Verborgenheit, an die Relativität und die Vieldeutigkeit der Gesundheit bleibt wichtig, wenn wir darüber nachdenken, wer welche Verantwortung für diese Gesundheit hat: das Individuum oder die Gesellschaft? Oder beide? Und dann wofür genau?

Eine tiefer liegende Frage ist damit offen: Wozu überhaupt Gesundheit? Ist tatsächlich die Herstellung und Erhaltung von Gesundheit das oberste Ziel der Medizin und des Gesundheitswesens?

Es scheint klar, dass Gesundheit sowohl eine öffentliche Aufgabe als auch eine Privatangelegenheit ist, kein Entweder-Oder. Gesundheit ist tatsächlich etwas, das uns persönlich betrifft und teilweise auch
Die andere Seite ist aber ebenso deutlich. Gesundheit spielt sich zwar wesentlich im Körper und in der Seele der Individuen ab, sie ist aber aus ganz verschiedenen Gründen auch eine öffentliche Aufgabe. Vielen der krankmachenden Faktoren kann nur mit kollektiven Massnahmen begegnet werden. Verkehrssicherheit, sauberes Trinkwasser, die Eindämmung von Umweltschadstoffen usw., also die primäre Prävention muss in vielen Bereichen öffentlich organisiert sein, wenn sie überhaupt funktionieren soll. Gesundheitsförderung beinhaltet allgemeine Aufklärung, kollektive Bewusstseinsbildung und Stärkungsmassnahmen, die von der öffentlichen Hand in gemeinschaftlicher Verantwortung viel besser initiiert werden können als von Privaten.

«Public health» ist ein bemerkenswerter Begriff: als ob die Öffentlichkeit gesund sein könnte, oder das 
Öffentliche. Gesund und krank im eigentlichen Sinn sind die Individuen. Das ist der Kern eines medizinischen Ethos, das sich von Ideologien einer «Volksgesundheit» distanziert. Das Ziel der Medizin und letztlich auch der öffentlichen Gesundheit ist das Wohl aller Individuen. Public health meint deshalb nicht «the public's health», sondern die organisierten gemeinschaftlichen Aktivitäten zur Prävention von Krankheiten und zur Förderung körperlicher und seelischer Gesundheit der Menschen. [1]

\section{Individualisierung und Subjektivität}

Wenn man nur etwas in die Geschichte zurückblickt, sieht man, dass die Abgrenzung dieser beiden Verantwortungsdomänen der Individuen und der Gesellschaften keineswegs stabil ist. Sie hängen mit der Entwicklung des Wohlfahrtsstaates zusammen. Mit dessen Etablierung hat sich in vielen westlichen Ländern die Auffassung durchgesetzt, dass die Sicherstellung gesunder Lebensbedingungen eine gesellschaftliche Aufgabe und sogar ein Grundrecht der Individuen sei. Seit den 1970er Jahren wurde dies jedoch zunehmend

\section{«Ein soziales Wohlbefinden ist eine der Bedingungen der individuellen Gesundheit. Wir sind nicht abgetrennt lebende, egoistische Subjekte, die unser individuelles Glück zu maximieren trachten»}

in Frage gestellt, und es setzte ein Prozess der Individualisierung der Gesundheitsverantwortung ein. John Knowles schrieb 1977 als damaliger Präsident der Rockefeller Foundation: «Prevention of disease means forsaking the bad habits which many people enjoy overeating, too much drinking, taking pills, staying up at night, engaging in promiscuous sex, driving too fast, and smoking cigarettes. ... The cost of sloth, gluttony, alcoholic intemperance, reckless driving, sexual frenzy, and smoking is now a national, and not an individual responsibility.... one man's freedom in health is another man's shackle in taxes and insurance premiums.» [2].

Der polemische Ton von Knowles wäre heute längst überflüssig geworden. Was heute stört, ist aber der moralische Ton. Die Überzeugung hat sich durchgesetzt, dass für Lifestyle-Entscheidungen die Individuen zuständig sind. Genetik könnte ein neuer Faktor werden. Vor ein paar Wochen konnte man lesen, dass eine britische Versicherungsgesellschaft ihren Kunden Prämienverbilligungen anbieten will, wenn sie sich genetisch auf Krankheitsdispositionen testen lassen, ohne dass sie die Ergebnisse offenlegen müssen. Gil Baldwin, Managing Director of Norwich Union Healthcare wurde so zitiert: «I'm not interested in knowing what your results are. I'm interested that you are interested in going for a screen, because only a madman would get information saying you're at risk of a heart attack and do nothing with that information» [3]. Die Erwartung ist, dass Menschen, wenn sie ihre spezifischen Krankheitsrisiken kennen, sich eigenverantwortlich gesundheitsverträglicher verhalten werden und deshalb der Versicherung weniger zur Last fallen. In einem individualisierten Regime der Prävention, das mit den Instrumenten der moralischen Selbstverantwortung arbeitet - und mit entsprechender Zuschreibung von Schuld nach unterlassenen Massnahmen -, ist die Offenlegung gar nicht nötig. Ich erwarte nicht, dass dies zu einer Gesundheitsdiktatur führt, auch nicht zur Individualisierung im Sinn der Vereinzelung, aber ich glaube, es wird doch zu einer Neuverteilung von Verantwortung führen, zu einem neuen Sozialvertrag sozusagen zwischen Einzelnen mit den privaten und den öffentlichen Institutionen. Die Individuen werden, wie es Nikolas Rose [4] ausdrückte, neue «obligations of genetic knowledge» erkennen müssen, auch wenn sie es selbst nicht wollen, eine scheinbar naturgegebene «genetische Verantwortlichkeit» und die Aufgabe einer «genetischen Klugheit» («genetic prudence») als in neuem Sinn «aktive» Bürgerinnen und Bürger.

Im Verhältnis von individuellem Wohl und einem guten Gelingen der Gemeinschaft geht es aber nicht nur um den Schutz der individuellen Freiheit gegenüber dem Einwirken des Kollektivs. Es genügt nicht, wenn wir nur sagen, dass das individuelle Wohl aus ethischer Sicht Priorität hat. Denn auch das Wohl der Einzelnen ist ja ganz wesentlich abhängig von der guten Vergesellschaftung. Ein soziales Wohlbefinden ist eine der Bedingungen der individuellen Gesundheit. Wir sind nicht abgetrennt lebende, egoistische Subjekte, die unser individuelles Glück zu maximieren trachten. Das ist ein Menschenbild, das heute zu Recht als «sozialer Atomismus» kritisiert wird. Subjektivität beinhaltet das Anerkannt-Werden. Das Ich entsteht nicht aus sich selbst heraus. Es ist kein Ausdruck eines «in uns» liegenden genetischen Programms. Subjektivität entsteht vielmehr aus der Intersubjektivität: Das Du ist kein alter ego, sondern genuin alter: eine Andere, ein Anderer. Gute Medizin - einschliesslich guter öffentlicher Gesundheitssorge - kann deshalb nur gelingen, wenn sie Kommunikation und Beziehungen als Teil der Menschen ansieht, nicht als ein Anhängsel oder gar als Komplikation.

\section{Beispiele aus der Arbeit der NEK-CNE}

Die Frage, wie die Verantwortung für die Gesundheit gesellschaftlich aufgeteilt werden kann, kam in verschiedenen Projekten der NEK-CNE vor. Ich möchte an ein paar Beispiele erinnern. Im Jahr 2001, als die NEK eingesetzt wurde, lag ein Antrag einer Genfer Forschergruppe Krause und Jaconi an den Schweizerischen Nationalfonds zu einem Projekt vor, das mit importierten embryonalen Stammzellen arbeiten sollte. Die Situation war hoch politisiert und die Medien überboten sich gegenseitig mit Heils- und Unheilsprognosen. Die Rechtslage in der Schweiz war un- 
klar. Das Fortpflanzungsmedizingesetz verbot die Entnahme einer Zelle aus einem Embryo, um daran Untersuchungen anzustellen - eine Bestimmung, die aber offensichtlich nicht gegen Stammzellenforschung, sondern gegen die Präimplantationsdiagnostik gerichtet war. Der Nationalfonds verschob die Entscheidung und forderte im Frühling 2001 eine öffentliche Diskussion über die rechtlichen und ethischen Implikationen. Nach den Sommerferien, während zwischenzeitlich aber die NEK-CNE eingesetzt worden war, stellte er sich auf den Standpunkt, dass die Debatte nun genügend Zeit gehabt habe, und schickte sich an, über die Bewilligung des Gesuchs zu entscheiden. implantationsdiagnostik auf sich zu nehmen und ein neues Kind zu zeugen. Die Kommission hat den heiklen Punkt darin gesehen, dass die Spenderinnen und Spender, bzw. die Eltern in eine Situation moralisch hoch aufgeladene Situation gestellt werden, in der sie fast keine Wahl haben. Daher ging es der Kommission weniger darum, ob und wann Lebendspende oder Retterbabys ethisch legitim sind, sondern darum, welche Rahmenbedingungen entstehen. Diese Rahmenbedingungen müssen sorgfältig überdacht werden, und zwar öffentlich, nicht nur bezüglich der Heilung in konkreten Fällen. Welche Bedingungen müssen erfüllt sein, um die Entscheidung der Spender oder der Eltern frei-

\section{«In diesem Fall ging es darum, der Politik eine Regelungshoheit (...) zu erhalten, gegenüber der Wissenschaft, die (...) die Forschung möglichst schnell voranbringen wollte»}

Bereits in ihrer ersten Sitzung entschloss sich die NEK deshalb zu einer Stellungnahme. In dieser beharrte sie aus ethischen Gründen auf dem Primat der Politik. Der Verlauf der Grenzen der Verfügbarkeit werdenden menschlichen Lebens für die Forschung, so sagte die Kommission, dürfe nicht den interessierten Parteien, also hier der Forschung, überlassen bleiben. Es handle sich um ein grundlegendes Rechtsproblem, das de lege ferenda diskutiert und vom Gesetzgeber erst geklärt werden muss. Solange nicht einmal die ethischen Implikationen verschiedener möglicher Regelungen klar seien, dürfe die Forschungsgemeinschaft in diesem Bereich kein Präjudiz schaffen. Sehr bald darauf hat das Parlament einen Entwurf zum Stammzellenforschungsgesetz verabschiedet, welcher Stammzellforschung innerhalb bestimmter Grenzen erlaubt und der 2004 nach einem Referendum sogar durch das Volk deutlich angenommen wurde.

In diesem Fall ging es darum, der Politik eine Regelungshoheit in einem rechtlichen Kernbereich zu erhalten, gegenüber der Wissenschaft, die mit Argumenten des medizinischen Fortschritts und mit therapeutischen Versprechen die Forschung möglichst schnell voranbringen wollte.

Zwei weitere Beispiele nehme ich aus dem Bereich Transplantationsmedizin. Die Lebendspende von Organen für Verwandte und das Retterkind, also die Erzeugung und Auslese eines Embryos, das die geeigneten immunologischen Marker trägt, damit das Kind für ein krankes Geschwister hämatopoietische Stammzellen geben kann. In beiden Fällen können mögliche Spender, die Familienangehörige retten können, oder die Eltern, die ihr krankes Kind retten können, in eine moralische no choice Situation kommen, in der sie vor ihrem Gewissen keine andere Wahl haben, als ein Organ zu spenden bzw. die Belastungen der IVF und Prä- willig werden zu lassen? Gibt es solche Bedingungen? Die Bedingungen müssten gewährleisten, dass sich darin ihre eigene Handlungsverantwortung und nicht ein systembedingter moralischer Zwang zum Ausdruck bringt. Die Kommission hat sich solche Bedingungen im Fall der Lebendspende vorstellen können und hat z.B. eine unabhängige Ombudsstelle vorgeschlagen, die allen Beteiligten offen steht. Im Fall des Retterkindes blieb sie gespalten.

In diesen Fällen ging es darum, das Thema der öffentlichen Verantwortung so herauszuarbeiten, dass sie die Entstehung einer freiheitlich qualifizierten individuellen Verantwortung ermöglicht und unterstützt. Das heisst, es ging darum, institutionelle Strukturen zu entwerfen, um eine individuelle ethische Entscheidung der Einzelnen gegenüber der situationsbedingten «Moral» in Schutz zu nehmen, d.h. gerade vor einem möglicherweise übermächtigen therapeutischen Imperativ.

Mein drittes Beispiel ist die organisierte Suizidbeihilfe. Hier hat die Kommission sehr sorgfältig darauf Acht gegeben, keine Bedingungen zur ethischen Legitimität von Beihilfehandlungen zu formulieren. Sie hat damit vielleicht manche Erwartungen enttäuscht. Sie tat dies aber ganz bewusst, weil es die klare Empfindung gab, dass es nicht die Aufgabe einer nationalen Ethikkommission sein kann, ethische Legitimitätsbedingungen für Selbsttötung festzulegen. Der Suizid wurde als «tragische Handlung» aufgefasst, die aus einer Situation der Hoffnungslosigkeit, Sinn- oder irgendeiner Ausweglosigkeit gewählt wird und sich dem rationalen Schema der Legitimität entzieht. Der ethisch heikle Punkt liegt gemäss der Analyse der NEK-CNE woanders: Es ist die Sorgfalt der Abklärung, ob einem Wunsch nach Suizidbeihilfe gefolgt werden soll oder ob für den betreffenden Menschen andere 
Möglichkeiten der Hilfe offen stehen sollten. Um diese Sorgfalt der Abklärung zu gewährleisten, hat die Kommission die staatliche Aufsicht über die organisierte Suizidhilfe vorgeschlagen und die Einführung von gesetzlichen Kriterien, welche der Vorbereitung einer Entscheidung den nötigen Raum, die nötige Zeit und die nötige Qualität gewähren.

Auch hier war ein fragiles Verhältnis von öffentlicher und privater Verantwortung das Thema. Die öffentliche Hand musste in ihre Verantwortung gerufen werden.

\section{Handlungsfähigkeit, erfülltes Leben}

Blicken wir auf die drei Beispiele Stammzellen, Lebendspende/Retterkind und Sterbehilfeorganisationen zurück. Mir fällt dreierlei auf. Erstens ist die Autonomie, die es in allen Fällen zu schützen galt, keine Autonomie im individualistischen Sinn, sondern immer eine Handlungsfähigkeit im Bezug auf Andere: Im ersten Fall war es die Verantwortung der demokratischen Politik. Im zweiten Fall war es die Selbstbestimmung der fürsorglich verantwortlichen Familienangehörigen oder der Eltern. Im dritten Fall war es die Handlungsfähigkeit des Sterbewilligen in seinem Lebenskontext und gleichzeitig die Mobilisierung von Mitverantwortung von denen, die vielleicht noch eine Möglichkeit haben, den Lebenskontext günstig zu verändern.

Die zweite Beobachtung bezieht sich auf die Ziele im Gesundheitswesen. Wie in der Medizin, ist das letzte Ziel nicht die Gesundheit selbst, sondern das Wohl der Menschen, die gesund und krank sein können. Das gilt auch für die Präventivmedizin und die Massnahmen der öffentlichen Gesundheitsförderung. Nicht die Gesundheit, welche uns sowieso verborgen ist, ist das oberste Ziel. Letztlich geht es um das erfüllte Leben der Betroffenen. Manchmal heisst ein erfülltes Leben auch, gelassen sein zu dürfen, eine nicht abwendbare Krankheit oder die Endlichkeit des Lebens annehmen zu können.

\section{«Ethik, wie sie die NEK-CNE versteht, ist immer eine}

Aufforderung zur Reflexion, eine Hilfe zum selber Sehen, selber Wahrnehmen und selber Denken gewesen»

Und drittens ist das Verhältnis zwischen individueller Zuständigkeit und kollektiver Verantwortung in allen drei Situationen je besonders gelagert. Einmal geht es um rechtliche Rahmenbedingungen, ein anderes Mal um ein sozio-technisch hergestelltes Arrangement von medizintechnischen Offerten, im dritten Fall war es eine Solidaritätspflicht der Gemeinschaft angesichts von semiprofessionalisierten Angeboten zur technischen Unterstützung bei der Selbsttötung, welche für Menschen, die suizidal sind, die Situation verändern.

\section{Was eine nationale Ethikkommission kann}

Eine politikberatende Nationale Ethikkommission kann in solchen brisanten Fragen für die Politik eine unabhängige, weltanschaulich breit abgestützte Referenz sein, die auf Augenhöhe mit den internationalen Fachdebatten arbeitet. Sie liefert nicht pfannenfertige Rezepte, wie man heikle Themen in der Schweiz moralisch korrekt über die Bühne bringen kann.

Die Analyse und die Beschreibung der Situationen und ihrer ethischen Implikationen, das Ausloten der Handlungsalternativen oder der Entwurf von Regelungsmodellen, also der deskriptiv-analytische Teil, sind der wichtigste Teil der Stellungnahmen der NEKCNE, wichtiger als ihre Empfehlungen, die am Schluss, manchmal nach Mehrheit und Minderheiten aufgefächert, herauskommen. So liefert die Kommission nämlich den Politikerinnen und Politikern eine Grundlage, um zu eigenen Schlüssen zu kommen. Ethik, wie sie die NEK-CNE versteht, ist immer eine Aufforderung zur Reflexion, eine Hilfe zum selber Sehen, selber Wahrnehmen und selber Denken gewesen, kein Aufschlucken von Verantwortung durch ein Gremium von staatlich approbierten Moralexperten. Sowohl die abweichenden wie auch die zustimmenden politischen Meinungen können robuster sein, wenn sie bezüglich der ethischen Implikationen besser abgestützt sind. Dazu sollen substantielle Vorarbeiten der Nationalen Ethikkommission beitragen

\section{Referenzen}

1 Petersen A, Bunton R. The New Genetics and the Public's Health. London/New York: Routledge; 2002: p. 73 ff.

2 Knowles JH. Responsibility of Individual. Daedalus. 1977:106(1): 57-80. Zit. nach Kollek R, Lemke T. 2008: p. 223f.

3 Henderson M. DNA scan could cut cost of insurance even if results kept secret. The Times. 28. März 2009.

4 Rose N. Will biomedicine transform society? The political economic, social and personal impact of medical advances in the twenty first century. BIOS working paper no. 1.2008. (www.lse.ac.uk/ collections/BIOS/workingpapers).

\section{Weitere Literatur zum Thema}

- Gadamer HG. Über die Verborgenheit der Gesundheit. Aufsätze und Vorträge. 2. Aufl. Frankfurt a. M. Suhrkamp;1993.

- Kollek R, Lemke T. Der medizinische Blick in die Zukunft. Frankfurt: Campus;2008.

- Rose N. Race, Risk and Medicine in the Age of «Your Own Personal Genome». BioSocieties. 2008;3:423-39.

- Rose N, Novas C. Biological Citizenship. In: Ong A, Collier S (eds.): Global Assemblages: Technology, Politics and Ethics as Anthropological Problems. Malden, MA: Blackwell;2005.

- WHO. Preamble to the Constitution of the World Health Organization as adopted by the International Health Conference. New York, 19-22 June, 1946; signed on 22 July 1946 by the representatives of 61 States (Official Records of the World Health Organization, no. 2, p. 100) and entered into force on 7 April 1948. 